K. Yamato

Nagoya Math. J.

Vol. 115 (1989), 87-104

\title{
ALGEBRAIC RIEMANN MANIFOLDS
}

\author{
KAZUO YAMATO
}

\section{Introduction}

In the present paper, we are concerned with the problem to know whether two algebraic Riemann manifolds are isometric or not, where we mean Riemann manifolds of class $C^{\Omega}$ (real algebraic smoothness or Nash category) simply by algebraic Riemann manifolds.

For this purpose we introduce notions of minimal differential polynomials and singular base points, both of which are isometry invariants. With the aid of these invariants, we give the following isometry theorem for algebraic Riemann manifolds:

MaIN TheOREM. Let $M, \bar{M}$ be connected, simply connected, complete, $C^{\Omega}$ Riemann manifolds of dimension $n \geqq 2$. Assume that the minimal differential polynomials of $M, \bar{M}$ coincide. Let $p \in M, \bar{p} \in \bar{M}$, and assume that $p, \bar{p}$ are not singular base points. If there exists a linear isometry $I: T_{p}(M) \rightarrow T_{\bar{p}}(\bar{M})$ which preserves the curvatures of $M, \bar{M}$ and their first $4 n-5$ covariant differentials, then $M$ and $\bar{M}$ are isometric by an isometry $h: M \rightarrow \bar{M}$ satisfying $h(p)=\bar{p},\left(h_{*}\right)_{p}=I$.

With this theorem we obtain a characterization of homogeneous Riemann manifolds:

TheOREM 3.4. Let $M$ be a connected, simply-connected, complete, $C^{\omega}$ Riemann manifolds of dimension $n \geqq 2$. Assume that $M$ is infinitesimally homogeneous of order $3 n-5$ in the sense that for any points $p, q$ of $M$ there exists a linear isometry $I: T_{p}(M) \rightarrow T_{q}(M)$ which preserves the curvature and its first $3 n-5$ covariant differentials. Then $M$ is homogeneous.

This theorem improves a result of Singer [10], which states that if $M$ is infinitesimally homogeneous of order $\frac{n(n-1)}{2}$, then $M$ is homogeneous.

Received May 19, 1987. 
Contrary to the $C^{\infty}$ or $C^{\omega}$ case, there exist $C^{\Omega}$ Riemann manifolds which admit no $C^{\Omega}$ function except constants. We give a criterion for the existence of sufficiently many $C^{\Omega}$ functions:

Theorem 4.2. Let $M$ be a compact $C^{\Omega}$ Riemann manifold. Assume that for any distinct points $p, q$ of $M$ there exists no isometry $h, h(p)=q$, of a neighborhood of $p$ onto a neighborhood of $q$. Then there exist $C^{\Omega}$ functions $f_{1}, \cdots, f_{N}$ on $M$ such that $\left(f_{1}, \cdots, f_{N}\right): M \rightarrow \boldsymbol{R}^{N}$ is an embedding (in the differential topological sense).

We also extend the Myers-Steenrod isometry theorem to the $C^{\Omega}$ case as follows:

CoRollary 4.4. Under the hypothesis of Theorem 4.2, an isometry between two $C^{\Omega}$ Riemann manifolds $M, \bar{M}$, if any, is of class $C^{\Omega}$.

We illustrate here minimal differential polynomials and singular base points. Roughly speaking, the minimal polynomial of $M$ is a polynomial $\phi_{M}=\phi_{M}\left(\xi_{0}, \cdots, \xi_{r}\right)$ such that for any geodesic $\gamma=\gamma(t)$ on $M$ and any Jacobi field $J$ along $\gamma$, the function $\xi(t)=\frac{1}{2}\|J(t)\|^{2}$ satisfies the differential equation $\phi_{M}\left(\xi, \xi^{\prime}, \cdots, \xi^{(r)}\right)=0$. A singular base point of $M$ is a point $q$ such that on the "fibre" over $q$, the differential equation $\phi_{M}\left(\xi, \cdots, \xi^{(r)}\right)=0$ can not be written in a normal form.

Then we are led to an intuitive proof of Proposition 2.1 and therefore of Main Theorem, as follows: Using the geodesic coordinates around $p, \bar{p}$ and the given linear mapping $I$, we can construct the (local) diffeomorphism $h$. We have to prove that $h$ is isometric. To do this, let $\gamma=\exp t X$ be a geodesic starting at $p$ in $M$, and let $\bar{\gamma}=\exp t I(X)$ be the corresponding geodesic in $\bar{M}$. We prove that for fixed $\tau, h_{*}: T_{r(\tau)} M \rightarrow T_{\bar{r}(\tau)} \bar{M}$ is isometric. Fix $V \in T_{r(\tau)} M$ and consider the Jacobi field $J$ along $\gamma$ such that $J(0)=0$, $J(\tau)=V$. Let $\bar{J}$ be the Jacobi field along $\bar{\gamma}$ such that $\bar{J}(0)=0, \bar{J}(\tau)=$ $h_{*} V$. The assumption $\phi_{M}=\phi_{\bar{M}}$ implies that the functions $\xi(t)=\frac{1}{2}\|J(t)\|^{2}$, $\bar{\xi}(t)=\frac{1}{2}\|\bar{J}(t)\|^{2}$ satisfy the same differential equation. Moreover, the existence of the linear mapping $I$ ensures that $\xi(t), \bar{\xi}(t)$ satisfy the same initial condition. Hence, by the uniqueness theorem for solutions of differential equations we can conclude that $\xi(t)=\bar{\xi}(t)$ and hence $\|V\|=$ $\left\|h_{*} V\right\|$, as desired. More accurately, we apply the uniqueness theorem to perturvations on $\xi, \bar{\xi}$ under the assumption of the nonsingularity of $p, \bar{p}$ and then we conclude that $\xi=\bar{\xi}$ from the continuous dependence on initial conditions. 
Our approach to the equivalence problem of Riemann manifolds sheds modern light on the classical argument of E. Cartan. In fact, by introducing the notion of minimal differential polynomial we regard the condition for the existence of isometries as an initial condition of the differential equation and hence we can reduce the proof of the isometry theorem to the uniqueness theorem for solutions of differential equations. This clarifies Cartan's argument in his isometry theorem.

Finally, I would like to thank Professor Shikata for his valuable suggestions for improving the exposition.

\section{§1. Minimal differential polynomials and singular base points}

We begin by recalling the notion of class $C^{\Omega}$. Let $U$ be an open subset of the $n$-dimensional real vector space $\boldsymbol{R}^{n}$. A function $f: U \rightarrow \boldsymbol{R}$ is said to be of class $C^{\Omega}$ if $f$ is of class $C^{\omega}$ (i.e. real analytic) and if for each connected component $U_{0}$ of $U$, there is a non-zero polynomial $P=$ $P\left(x_{1}, \cdots, x_{n+1}\right)$ in $n+1$ variables with real coefficients such that $P\left(x_{1}, \cdots, x_{n}\right.$, $\left.f\left(x_{1}, \cdots, x_{n}\right)\right)=0$ on $U_{0}$. After the definition of function of class $C^{\Omega}$, we have the notion of manifold of class $C^{\Omega}$ in the usual way (cf. [8], [9]). The vector space $\boldsymbol{R}^{n}$ has the natural $C^{\Omega}$ structure such that polynomial functions on $\boldsymbol{R}^{n}$ are of class $C^{\Omega}$. We always equip $\boldsymbol{R}^{n}$ with this $C^{\Omega}$ structure, unless otherwise specified.

Furthermore, we can define tensor fields of class $C^{\Omega}$ (on $C^{\Omega}$ manifolds) in the obvious way. Especially, a Riemann manifold of class $C^{\Omega}$ (or simply a $C^{\Omega}$ Riemann manifold) is a manifold of class $C^{\Omega}$ with a $C^{\Omega}$ Riemann metric.

To introduce the notion of minimal differential polynomial we recall some definitions. First, $\boldsymbol{R}\left[\xi_{0}, \cdots, \xi_{r}\right]$ denotes the ring of polynomials in variables $\xi_{0}, \cdots, \xi_{r}$ with real coefficients. Let $P \in R\left[\xi_{0}, \cdots, \xi_{r}\right]$. Suppose that $P$ is written as

$$
P=c_{p_{0} \cdots p_{r}} \xi_{0}^{p_{0}} \cdots \xi_{r}^{p_{r}}+\sum_{\left(q_{0}, \cdots, q_{r}\right)<\left(p_{0}, \cdots, p_{r}\right)} c_{q_{0} \cdots q_{r}} \xi_{0}^{q_{0}} \cdots \xi_{r}^{q_{r}}, c_{p_{0} \cdots p_{r}} \neq 0, \quad p_{r} \geqq 1,
$$

where $\left(q_{0}, \cdots, q_{r}\right)<\left(p_{0}, \cdots, p_{r}\right)$ means that $q_{i}<p_{i}$ for the largest integer $i(0 \leqq i \leqq r)$ such that $p_{i} \neq q_{i}$. Then we call $r$ the order of $P$. If $c_{p_{0} \cdots p_{r}}$ $=1$, then we shall say that $P$ is monic.

Next, let $\gamma=\gamma(t)$ be a geodesic in a Riemann manifold. Denote by $\dot{\gamma}(t)$ the vector tangent to $\gamma$ at $\gamma(t)$. For a vector field $Y$ along $\gamma$, we denote by $Y^{\prime}(t)$ the covariant derivative $\nabla_{\dot{r}(t)} Y$ of $Y$ in the direction of 
$\dot{\gamma}(t)$. We denote by $\langle$,$\rangle the Riemann metric, and by \|\|$ the norm of tangent vectors.

Definition 1.1. Let $M$ be a connected $C^{\infty}$ Riemann manifold of dimension $n \geqq 2$. Let $\phi \in R\left[\xi_{0}, \cdots, \xi_{r}\right]$ be an irreducible, monic polynomial with order $r$. We shall say that $\phi$ is the minimal differential polynomial of $M$ if the following conditions are satisfied:

(i) For any geodesic $\gamma$ with $\|\dot{\gamma}(t)\|=1$ and for any Jacobi field $J$ along $\gamma$ with $\langle\dot{\gamma}(t), J(t)\rangle=0$, the function $\xi(t)=\frac{1}{2}\langle J(t), J(t)\rangle$ satisfies the differential equation

$$
\phi\left(\xi(t), \xi^{\prime}(t), \cdots, \xi^{(r)}(t)\right)=0,
$$

where $\xi^{(i)}(t)=\left(d^{i} / d t^{i}\right) \xi(t)$.

(ii) If an irreducible, monic polynomial $\psi \in \boldsymbol{R}\left[\xi_{0}, \cdots, \xi_{s}\right]$ with order $s$, satisfies $\psi\left(\xi(t), \cdots, \xi^{(s)}(t)\right)=0$ for every $\xi(t)$ as in (i), then we have $s=r$ and $\phi=\psi$, or we have $s>r$.

From the definition we see that the minimal differential polynomial, if it exists, is uniquely determined.

Definition 1.2. Suppose that $M$ has a minimal differential polynomial $\phi\left(\xi_{0}, \cdots, \xi_{r}\right)$ of order $r$. We shall say that $p \in M$ is a singular base point of $\phi$ if for any unit tangent vector $X$ at $p$ and any Jacobi field $J$ along $\gamma(t)=\exp t X$ with $\langle\dot{\gamma}(t), J(t)\rangle=0$, we have

$$
\frac{\partial \phi}{\partial \xi_{r}}\left(\xi(0), \xi^{\prime}(0), \cdots, \xi^{(r)}(0)\right)=0
$$

where $\xi(t)=\frac{1}{2}\langle J(t), J(t)\rangle$.

Lemma 1.3. Let $M$ be a connected $C^{\Omega}$ Riemann manifold of dimension $n \geqq 2$. Then there exists the minimal differential polynomial $\phi$ of $M$. The order of $\phi$ is equal to or less than $4 n-3$. The set of singular base point of $\phi$ is closed and nowhere dense in $M$.

To prove Lemma 1.3 we need a lemma.

LEMMA 1.4. Let $M$ be a $C^{\infty}$ Riemann manifold of dimension $n \geqq 2$. Let $G(M)$ be the $C^{\infty}$ submanifold of the Whitney sum $T(M) \oplus T(M) \oplus T(M)$, defined by

$$
\begin{gathered}
G(M)=\{(X, Y, Z) \in T(M) \oplus T(M) \oplus T(M) \mid\langle X, X\rangle=1, \\
\langle X, Y\rangle=\langle X, Z\rangle=0\} .
\end{gathered}
$$


For a point $p$ of $M$, let $G_{p}(M)$ be the fibre over $p$, i.e. the set of triples $(X, Y, Z)$ in $G(M)$ whose base point is $p$. For $(X, Y, Z) \in G(M)$, denote by $J_{X, Y, Z}$ the Jacobi field along $\exp t X$ with $J_{X, Y, Z}(0)=Y, J_{X, Y, Z}^{\prime}(0)=Z$. For each non-negative integer $k$, define a $C^{\infty}$ function $g_{k}: G(M) \rightarrow R$ by the formula

$$
g_{k}(X, Y, Z)=\xi^{(k)}(0)
$$

where $\xi^{(k)}(0)$ is the $k$-th derivative at $t=0$ of the function $\xi(t)=\frac{1}{2}\left\langle J_{X, Y, Z}(t)\right.$, $\left.J_{X, Y, Z}(t)\right\rangle$.

Then the functions $g_{k}$ have the following properties:

(i) For any geodesic $\gamma$ with $\|\dot{\gamma}(t)\|=1$ and any Jacobi field $J$ along $\gamma$ with $\langle\dot{\gamma}(t), J(t)\rangle=0$, the $k$-th derivatives $\xi^{(k)}(t)$ of the function $\xi(t)=$ $\frac{1}{2}\langle J(t), J(t)\rangle$ are given by the formulas

$$
\xi^{(k)}(t)=g_{k}\left(\dot{\gamma}(t), J(t), J^{\prime}(t)\right)
$$

(ii) For each point $p$ of $M$, the restrictions $\left.g_{k}\right|_{G_{p}(M)}: G_{p}(M) \rightarrow R$ are expressed as restrictions of some polynomial functions $T_{p}(M) \oplus T_{p}(M) \oplus T_{p}(M)$ $\rightarrow \boldsymbol{R}$. Hence in particular, $\left.g_{k}\right|_{G_{p}(M)}$ are $C^{\Omega}$ functions on the $C^{\Omega}$ manifold $G_{p}(M)$.

(iii) If $M$ is a $C^{\Omega}$ Riemann manifold, then $g_{k}$ are $C^{\Omega}$ functions on the $C^{s}$ manifold $G(M)$.

(iv) Let $\bar{M}$ be another $C^{\infty}$ Riemann manifold of dimension $n$, and define $\bar{g}_{k}: G(\bar{M}) \rightarrow R, k=0,1, \cdots$, in the same way as above. Let $p \in M$, $\bar{p} \in \bar{M}$, and let $k$ be fixed. Assume that there exists a linear isometry $I: T_{p}(M) \rightarrow T_{\bar{p}}(\bar{M})$ such that for any non-negative integer $l \leqq k-2$, the l-th covariant differentials $\nabla^{\prime} R, \nabla^{\prime} \bar{R}$ of the curvature tensors $R, \bar{R}$ of $M, \bar{M}$ are preserved by $I$, i.e. the relation

$$
\left\langle\left(\nabla^{l} R\right)\left(X, Y ; Z_{1} ; \cdots ; Z_{l}\right) V, W\right\rangle=\left\langle\left(\nabla^{\imath} \bar{R}\right)\left(\bar{X}, \bar{Y} ; \bar{Z}_{1} ; \cdots ; \bar{Z}_{l}\right) \bar{V}, \bar{W}\right\rangle
$$

holds for any $X, Y, V, W, Z_{1}, \cdots, Z_{l} \in T_{p}(M)$, where $\bar{X}=I(X), \cdots, \bar{Z}_{l}=I\left(Z_{l}\right)$, and $\nabla^{\circ} R=R, \nabla^{0} \bar{R}=\bar{R}$. Then we have

$$
g_{k}(X, Y, Z)=\bar{g}_{k}(I(X), I(Y), I(Z))
$$

for any $(X, Y, Z) \in G_{p}(M)$.

Proof. Part (i) is an immediate consequence of the fact that $J_{\dot{\gamma}^{(t), J(t), J^{\prime}(t)}}(s)=J(t+s)$. To prove the rest, let $(X, Y, Z) \in G(M)$ and put $\gamma=\exp t X, J=J_{X, Y, Z}$. Then for the function $\xi(t)=\frac{1}{2}\langle J(t), J(t)\rangle$ of $t$, it 
is clear that $\xi^{\prime}(t)=\left\langle J^{\prime}(t), J(t)\right\rangle$. Since $J^{\prime \prime}(t)=R(\dot{\gamma}(t), J(t)) \dot{\gamma}(t)$, omitting the parameter $t$ for simplicity, we have

$$
\xi^{\prime \prime}=\langle R(\dot{\gamma}, J) \dot{\gamma}, J\rangle+\left\langle J^{\prime}, J^{\prime}\right\rangle .
$$

Using the covariant differential $\nabla R$ of $R$ and the symmetry property of the operator $R(\dot{\gamma}, *) \dot{r}$ we have

$$
\xi^{\prime \prime \prime}=\langle(\nabla R)(\dot{\gamma}, J ; \dot{\gamma}) \dot{\gamma}, J\rangle+4\left\langle R\left(\dot{\gamma}, J^{\prime}\right) \dot{\gamma}, J\right\rangle .
$$

Generally, for $k \geqq 4$ the function $\xi^{(k)}(t)$ can be expressed as

$$
\begin{aligned}
\xi^{(k)}= & \sum_{\lambda} A_{\lambda}\left(R, \cdots, \nabla^{k-2} R\right)(\underbrace{\dot{\gamma}, \ldots, \dot{\gamma}}_{k \text { times }}, J, J) \\
& +\sum_{\mu} B_{\mu}\left(R, \cdots, \nabla^{k-3} R\right)(\underbrace{\dot{\gamma}, \ldots, \dot{\gamma}}_{k-1 \text { times }}, J, J^{\prime}) \\
& +\sum_{\nu} C_{\nu}\left(R, \cdots, \nabla^{k-4} R\right)(\underbrace{\dot{\gamma}, \cdots,}_{k-2 \text { times }}, J^{\prime}, J^{\prime}),
\end{aligned}
$$

where $A_{\lambda}\left(R, \cdots, \nabla^{k-2} R\right)$ are certain tensor fields on $M$, of type $(0, k+2)$, obtained by contractions of tensor products of $R, \cdots, V^{k-2} R$, and $B_{\mu}(R, \cdots$, $\left.\nabla^{k-3} R\right), C_{2}\left(R, \cdots, V^{k-4} R\right)$ are also tensor fields of the same sort. Note that the tensor fields $A_{\lambda}, B_{\mu}, C_{\nu}$ are defined independent of the choice of $(X, Y, Z) \in G(M)$. Putting $t=0$ shows that $g_{k}, k=0,1, \ldots$, are expressed as

$$
\begin{aligned}
& g_{0}(X, Y, Z)=\frac{1}{2}\langle Y, Y\rangle, \\
& g_{1}(X, Y, Z)=\langle Y, Z\rangle, \\
& g_{2}(X, Y, Z)=\langle R(X, Y) X, Y\rangle+\langle Z, Z\rangle, \\
& g_{3}(X, Y, Z)=\langle(\nabla R)(X, Y ; X) X, Y\rangle+4\langle R(X, Z) X, Y\rangle,
\end{aligned}
$$

and for $k \geqq 4$

$$
\begin{aligned}
g_{k}(X, Y, Z)= & \sum_{\lambda} A_{\lambda}\left(R, \cdots, \nabla^{k-2} R\right)(X, \cdots, X, Y, Y) \\
& +\sum_{\mu} B_{\mu}\left(R, \cdots, \nabla^{k-3} R\right)(X, \cdots, X, Y, Z) \\
& +\sum_{\nu} C_{\nu}\left(R, \cdots, \nabla^{k-4} R\right)(X, \cdots, X, Z, Z) .
\end{aligned}
$$

From these formulas we verify at once our assertions (ii), (iii), (iv).

Proof of Lemma 1.3. Let $g_{k}: G(M) \rightarrow \boldsymbol{R}$ be the $C^{\Omega}$ functions defined in Lemma 1.4. Clearly, $G(M)$ is connected $(n \geqq 2)$, and $g_{0}, g_{1}$ are algebraically independent over $\boldsymbol{R}$. Since the dimension of $G(M)$ is $4 n-3$, 
some point of $G(M)$ but $d g_{0} \wedge \cdots \wedge d g_{r}=0$ on $G(M)$. Then we can find an irreducible polynomial $P \in R\left[\xi_{0}, \cdots, \xi_{r}\right]$ such that $\operatorname{deg}_{\xi_{r}} P \geqq 1$ and $P\left(g_{0}, \cdots, g_{r}\right)=0$ on $G(M)$. Dividing $P$ by the "leading coefficient", if $P$ is not monic, we get the desired polynomial $\phi$. Indeed, it is clear that the order of $\phi$ is $r \leqq 4 n-3$. To verify that $\phi$ satisfies condition (i) of Definition 1.1 , let $J$ be a Jacobi field along a geodesic $r$ with $\|\dot{\gamma}(t)\|=1$, such that $\langle\dot{\gamma}(t), J(t)\rangle=0$. Then $\left(\dot{\gamma}(t), J(t), J^{\prime}(t)\right) \in G(M)$ for any $t$. Hence we have

$$
\phi\left(g_{0}\left(\dot{\gamma}(t), J(t), J^{\prime}(t)\right), \cdots, g_{r}\left(\dot{\gamma}(t), J(t), J^{\prime}(t)\right)\right)=0
$$

and thus, by (i) of Lemma 1.4, we have the required relation

$$
\phi\left(\xi(t), \cdots, \xi^{(r)}(t)\right)=0 .
$$

Next, as for condition (ii) in Definition 1.1, it is clear that such a polynomial $\psi$ satisfies $\psi\left(g_{0}, \cdots, g_{s}\right)=0$ on $G(M)$. Since $g_{0}, \cdots, g_{r-1}$ are algebraically independent over $R$, we have $r \leqq s$. If $r=s$, then the irreducibilities of $\phi, \psi$ imply that $\phi=\psi$. The former part of our assertion is proved.

It remains to study the set $S$ of singular base points of $\phi$. We shall prove that its complement $S^{c}$ is open and dense in $M$. From Lemma 1.4 we know that a point $p$ of $M$ is a singular base point of $\phi$ if and only if

$$
\frac{\partial \phi}{\partial \xi_{r}}\left(g_{0}(X, Y, Z), \cdots, g_{r}(X, Y, Z)\right)=0 \text { for any }(X, Y, Z) \in G_{p}(M) \text {. }
$$

Hence the complement $S^{c}$ coincides with the image $\pi\left(\Sigma^{c}\right)$ of the set $\Sigma^{c}=\left\{(X, Y, Z) \in G(M) \mid \frac{\partial \phi}{\partial \xi_{r}}\left(g_{0}(X, Y, Z), \cdots, g_{r}(X, Y, Z)\right) \neq 0\right\}$ by the projection $\pi: G(M) \rightarrow M$. Since $\frac{\partial \phi}{\partial \xi_{r}}$ is a non-zero polynomial and $\phi$ is minimal in the sense of condition (ii) of Definition 1.1, we observe that the $C^{\Omega}$ function $\frac{\partial \phi}{\partial \xi_{r}}\left(g_{0}, \cdots, g_{r}\right): G(M) \rightarrow R$ is not a zero function. Therefore the set $\Sigma^{c}$ is open and dense in $G(M)$, and hence its image $\pi\left(\Sigma^{c}\right)$ is also open and dense in $M$, as desired. Lemma 1.3 is proved.

From Lemma 1.4 we have also:

Lemma 1.5. Assume that a connected $C^{\infty}$ Riemann manifold $M$ has a minimal differential polynomial $\phi\left(\xi_{0}, \cdots, \xi_{r}\right)$ of order $r$. Let $p \in M$, and let $G_{p}(M), g_{k}$ be as in Lemma 1.4. Denote by $\Sigma_{p}$ the set of triples $(X, Y, Z)$ $\in G_{p}(M)$ such that 


$$
\frac{\partial \phi}{\partial \xi_{r}}\left(g_{0}(X, Y, Z), \cdots, g_{r}(X, Y, Z)\right)=0 .
$$

Then $p$ is a singular base point of $\phi$ if and only if $\Sigma_{p}=G_{p}(M)$. If $p$ is not a singular base point of $\phi$ and if a triple $(X, Y, Z)$ lies in $\Sigma_{p}$, then there exists a sequence $\left\{\left(X_{i}, Y_{i}, Z_{i}\right)\right\}$ in $G_{p}(M)$ such that $\left(X_{i}, Y_{i}, Z_{i}\right) \notin \Sigma_{p}$ for any $i$ and $(X, Y, Z)=\lim _{i \rightarrow \infty}\left(X_{i}, Y_{i}, Z_{i}\right)$.

Proof. The former part is an immediate consequence of the definition of singular base point. To verify the latter part, we note that $\Sigma_{p}$ is an algebraic set in $G_{p}(M)$, by (ii) of Lemma 1.4. Hence if $p$ is not a singular base point of $\phi$, then in particular $\Sigma_{p}$ is closed and nowhere dense in $G_{p}(M)$. This proves our assertion.

\section{§ 2. The isometry theorems}

We can now state the local isometry theorem.

Proposition 2.1. Let $M, \bar{M}$ be connected, $C^{o}$ Riemann manifolds of dimension $n \geqq 2$. Assume that $M, \bar{M}$ have the same minimal differential polynomial $\phi$. Let $r$ be the order of $\phi$. Let $p \in M, \bar{p} \in \bar{M}$, and assume that both $p, \bar{p}$ are not singular base points of $\phi$. Assume that there exists a linear isometry $I: T_{p}(M) \rightarrow T_{\bar{p}}(\bar{M})$ which preserves the curvatures $R, \bar{R}$ of $M, \bar{M}$ and their first $r-2$ covariant differentials, i.e. satisfies the relations

$$
\begin{array}{r}
\left\langle\left(V^{k} R\right)\left(X, Y ; Z_{1} ; \cdots ; Z_{k}\right) V, W\right\rangle=\left\langle\left(V^{k} \bar{R}\right)\left(\bar{X}, \bar{Y} ; \bar{Z}_{1} ; \cdots ; \bar{Z}_{k}\right), \bar{V}, \bar{W}\right\rangle, \\
0 \leqq k \leqq r-2,
\end{array}
$$

for any $X, Y, \cdots, Z_{k} \in T_{p}(M)$, where $\bar{X}=I(X), \cdots, \bar{Z}_{k}=I\left(Z_{k}\right)$. Then there exists a $C^{\omega}$ isometry $h$ of a neighborhood of $p$ onto a neighborhood of $\bar{p}$ such that $h(p)=\bar{p}$, and the induced mapping $\left(h_{*}\right)_{p}$ at $p$ coincides with $I$.

By a standard argument on simply-connectedness (see, for example, [5, Vol. I, p. 256]), we get immediately the following global result.

Theorem 2.2. Let $M, \bar{M}$ be connected, simply connected, complete, $C^{\Omega}$ Riemann manifolds of dimension $n \geqq 2$. Assume that the minimal differential polynomials of $M, \bar{M}$ coincide. Let $p \in M, \bar{p} \in \bar{M}$, and assume that both $p, \bar{p}$ are not singular base points. If there exists a linear isometry $I: T_{p}(M) \rightarrow T_{\bar{p}}(\bar{M})$ which preserves the curvatures of $M, \bar{M}$ and their first $r-2$ covariant differentials, where $r$ is the order of the minimal differential 
$h(p)=\bar{p}$ and $\left(h_{*}\right)_{p}=I$.

Combining Lemma 1.3 and Theorem 2.2, we obtain

MAIN Theorem. Under the hypotheses of Theorem 2.2, if there exists a linear isometry $I: T_{p}(M) \rightarrow T_{\bar{p}}(\bar{M})$ which preserves the curvatures of $M, \bar{M}$ and their first $4 n-5$ covariant differentials, then $M$ and $\bar{M}$ are isometric by an isometry $h$ satisfying $h(p)=\bar{p},\left(h_{*}\right)_{p}=I$.

We can not expect that the isometry $h$ in the above proposition or theorems is of class $C^{\Omega}$. In $\S 4$, however, we shall prove that under a reasonable assumption, $h$ is of class $C^{a}$.

Proof of Proposition 2.1. Define a $C^{\omega}$ diffeomorphism $h$ of a neighborhood $U(p)$ of $p$ onto a neighborhood $\bar{U}(p)$ of $\bar{p}$ by the formula

$$
h(\exp t X)=\exp t I(X)
$$

for $x \in T_{p}(M),\|X\|=1$ and for small $|t|$. To prove that $h$ is an isometry, let $q \in U(p), V \in T_{q}(M)$, and write $q=\exp \tau X$ with $X \in T_{p}(M),\|X\|=1$, $\tau \in \boldsymbol{R}$. We have to prove $\langle V, V\rangle=\left\langle h_{*} V, h_{*} V\right\rangle$. To do this, we may assume that $\left\langle V,\left.\frac{d}{d t}\right|_{t=\tau} \exp t X\right\rangle=0$, because $h_{*}$ maps clearly the unit tangent vector $\left.\frac{d}{d t}\right|_{t=\tau} \exp t X$ to the unit tangent vector $\left.\frac{d}{d t}\right|_{t=\tau} \exp t I(X)$, and maps the orthogonal complement of $\left.\frac{d}{d t}\right|_{t=\tau} \exp t X$ to that of $\left.\frac{d}{d t}\right|_{t=\tau} \exp t I(X)$ (cf. [4, p. 8], [5, Vol. I, p. 165]). Let $J$ be the Jacobi field along exp $t X$ such that $J(0)=0, J(\tau)=V$ and hence $\left\langle J^{\prime}(0), X\right\rangle=0$. Recalling the fact that a Jacobi field is the infinitesimal variation of a family of geodesics, we observe that

$$
\begin{aligned}
h_{*} J(t) & =\left.h_{*} \frac{\partial}{\partial s}\right|_{s=0} \exp t\left(X+s J^{\prime}(0)\right) \\
& =\left.\frac{\partial}{\partial s}\right|_{s=0} h\left(\exp t\left(X+s J^{\prime}(0)\right)\right) \\
& =\left.\frac{\partial}{\partial s}\right|_{s=0} \exp t\left(I(X)+s I\left(J^{\prime}(0)\right)\right) \\
& =\bar{J}(t),
\end{aligned}
$$

where $\bar{J}$ is the Jacobi field along $\exp t I(X)$ such that $\bar{J}(0)=0, \bar{J}^{\prime}(0)=$ $I\left(J^{\prime}(0)\right)$. We contend that $\langle J(t), J(t)\rangle=\langle\bar{J}(t), \bar{J}(t)\rangle$. Putting $t=\tau$ will prove $\langle V, V\rangle=\left\langle h_{*} V, h_{*} V\right\rangle$. 
We consider the functions $\xi(t)=\frac{1}{2}\langle J(t), J(t)\rangle, \bar{\xi}(t)=\frac{1}{2}\langle\bar{J}(t), \bar{J}(t)\rangle$. To prove $\xi=\bar{\xi}$ we note that $\xi$, $\bar{\xi}$ satisfy the same differential equation

$$
\phi\left(\xi, \cdots, \xi^{(r)}\right)=\phi\left(\bar{\xi}, \cdots, \bar{\xi}^{(r)}\right)=0
$$

and the same initial conditions

$$
\xi^{(k)}(0)=\bar{\xi}^{(k)}(0), \quad k=0,1, \cdots, r
$$

(by (iv) of Lemma 1.4). Hence if the condition $\frac{\partial \phi}{\partial \xi_{r}}\left(\xi(0), \cdots, \xi^{(r)}(0)\right) \neq 0$ is satisfied, then by uniqueness theorem of solutions of differential equations we conclude that $\xi(t)=\bar{\xi}(t)$ for small $|t|$, and hence $\xi(t)=\bar{\xi}(t)$ for any $t$, because $\xi, \bar{\xi}$ are of class $C^{\omega}$.

In the case $\frac{\partial \phi}{\partial \xi_{r}}\left(\xi(0), \cdots, \xi^{(r)}(0)\right)=0$ we proceed as follows. With the notation in Lemma 1.5 we see that the condition $\frac{\partial \phi}{\partial \xi_{r}}\left(\xi(0), \cdots, \xi^{(r)}(0)\right)=0$ is equivalent to the condition $\left(X, 0, J^{\prime}(0)\right) \in \Sigma_{p}$. Then by the latter part of Lemma 1.5 we find a sequence $\left\{\left(X_{i}, Y_{i}, Z_{i}\right)\right\}$ in $G_{p}(M)$ such that $\left(X_{i}, Y_{i}, Z_{i}\right)$ $\notin \Sigma_{p}$ for any $i$ and $\left(X, 0, J^{\prime}(0)\right)=\lim _{i \rightarrow \infty}\left(X_{i}, Y_{i}, Z_{i}\right)$. It is clear that the corresponding sequence $\left\{\left(I\left(X_{i}\right), I\left(Y_{i}\right), I\left(Z_{i}\right)\right)\right\}$ in $G_{p}(\bar{M})$ also satisfies $\left(I\left(X_{i}\right), I\left(Y_{i}\right), I\left(Z_{i}\right)\right) \notin \bar{\Sigma}_{\bar{p}}$ for any $i$ because of Lemma 1.4, (iv), and that $\left(I(X), 0, \bar{J}^{\prime}(0)\right)=\lim _{i \rightarrow \infty}\left(I\left(X_{i}\right), I\left(Y_{i}\right), I\left(Z_{i}\right)\right)$. For each $i$, we let $J_{i}$ (resp. $\bar{J}_{i}$ ) the Jacobi field along $\exp t X_{i}$ with $J_{i}(0)=Y_{i}, J_{i}^{\prime}(0)=Z_{i}$ (resp. along $\exp t I\left(X_{i}\right)$ with $\left.\bar{J}_{i}(0)=I\left(Y_{i}\right), \bar{J}_{i}^{\prime}(0)=I\left(Z_{i}\right)\right)$, and consider the two functions $\xi_{i}(t)=\frac{1}{2}\left\langle J_{i}(t), J_{i}(t)\right\rangle, \bar{\xi}_{i}(t)=\frac{1}{2}\left\langle\bar{J}_{i}(t), \bar{J}_{i}(t)\right\rangle$. Then for each $i$, by the same reason as before we get $\xi_{i}(t)=\bar{\xi}_{i}(t)$. On the other and, by continuity of solutions on initial conditions we observe that for each $t$,

$$
J(t)=\lim _{i \rightarrow \infty} J_{i}(t), \quad \bar{J}(t)=\lim _{i \rightarrow \infty} \bar{J}_{i}(t),
$$

and hence

$$
\xi(t)=\lim _{i \rightarrow \infty} \xi_{i}(t), \quad \bar{\xi}(t)=\lim _{i \rightarrow \infty} \bar{\xi}_{i}(t) .
$$

Thus we conclude that $\xi(t)=\bar{\xi}(t)$. Our proposition is proved.

\section{§ 3. Homogeneous Riemann manifolds}

The proof of Proposition 2.1 shows

Proposition 3.1. Let $M, \bar{M}$ be connected, $C^{\omega}$ Riemann manifolds of dimension $n \geqq 2$. Assume that $M, \bar{M}$ have minimal differential polynomials 
$\phi, \bar{\phi}$ and that $p \in M, \bar{p} \in \bar{M}$ are not singular base points of $\phi, \bar{\phi}$, respectively. If $\phi$ and $\bar{\phi}$ coincide, and if there exists a linear isometry $I: T_{p}(M) \rightarrow T_{\bar{p}}(\bar{M})$ which preserves the curvatures of $M, \bar{M}$ and their first $r-2$ covariant differentials, where $r$ is the order of $\phi=\bar{\phi}$, then there exists a $C^{\omega}$ isometry $h$ of a neighborhood of $p$ onto a neighborhood of $\bar{p}$ such that $h(p)=\bar{p}$ and $\left(h_{*}\right)_{p}=I$.

The proof of Lemma 1.3 also shows

Lemma 3.2. If a connected $C^{\omega}$ Riemann manifold $M$ of dimension $n \geqq 2$ has a minimal differential polynomial $\phi$, then the set of singular base points of $\phi$ is closed and nowhere dense in $M$.

The following proposition implies that locally homogeneous, connected $C^{\infty}$ Riemann manifolds have minimal differential polynomials.

Proposition 3.3. Let $M$ be a connected, $C^{\infty}$ Riemann manifold of dimension $n \geqq 2$. Assume that for any two points $p$, $q$ of $M$, there exists a linear isometry $I: T_{p}(M) \rightarrow T_{q}(M)$ which preserves the curvature and its first $3 n-5$ covariant differentials. Then $M$ has a minimal differential polynomial $\phi$. The order of $\phi$ is equal to or less than $3 n-3$, and $\phi$ has no singular base point.

Proof. Fix $p \in M$, and consider the $C^{\Omega}$ function $\left.g_{k}\right|_{a_{p}(M)}: G_{p}(M) \rightarrow R$ as in (ii) of Lemma 1.4. Since the dimension of $G_{p}(M)$ is $3 n-3$, there exists an integer $r, 2 \leqq r \leqq 3 n-3$, such that $\left.g_{0}\right|_{a_{p}(M)}, \cdots,\left.g_{r-1}\right|_{a_{p}(M)}$ are algebraically independent (over $\boldsymbol{R}$ ), but $\left.g_{0}\right|_{a_{p}(M)}, \cdots,\left.g_{r}\right|_{a_{p}(M)}$ are algebraically dependent. Then we have an irreducible, monic polynomial $\phi \in \boldsymbol{R}\left[\xi_{0}, \cdots, \xi_{r}\right]$ such that $\operatorname{deg}_{\xi_{r}} \phi \geqq 1$ and $\phi\left(g_{0}, \cdots, g_{r}\right)=0$ on $G_{p}(M)$. We contend that $\phi\left(g_{0}, \cdots, g_{r}\right)=0$ on $G(M)$. This will prove that $\phi$ is the minimal differential polynomial of $M$. To prove our contention, let $q \in M$. It suffices to verify that $\phi\left(g_{0}, \cdots, g_{r}\right)=0$ on $G_{q}(M)$. This is an immediate consequence of the existence of the isometry $I: T_{p}(M) \rightarrow T_{q}(M)$ preserving the covariant differentials $\nabla^{k} R, k=0, \cdots, 3 n-5$, of the curvature $R$, and (iv) of Lemma 1.4. Clearly, there is no singular base point of $\phi$. Proposition 3.3 is proved.

Combining Propositions 3.1 and 3.2 and using simply-connectedness as before, we have at once the following characterization of homogeneous Riemann manifolds. 
Theorem 3.4. Let $M$ be a connected, simply connected, complete, $C^{\omega}$ Riemann manifold of dimension $n \geqq 2$. Assume that $M$ is infinitesimally homogeneous of order $3 n-5$ in the sense that for any $p, q \in M$, there exists a linear isometry $I: T_{p}(M) \rightarrow T_{q}(M)$ which preserves the curvature and its first $3 n-5$ covariant differentials. Then $M$ is homogeneous, i.e. for any $p, q \in M$, there exists a $C^{\omega}$ isometry $h$ of $M$ onto $M$ itself such that $h(p)=q$.

Remark 3.5. We mention the difference between Singer [10] and ours. Singer develops his argument on the bundle of orthonormal frames and hence he can discuss the Lie algebra structure of Killing vector fields. (Nomizu gives more algebraic treatment in [7].) On the other hand, we consider $G(M)$, the bundle of "Jacobi fields", in order to deal with the minimal differential polynomial. We can not discuss directly the structure of Killing vector fields, but we rather wish that we could characterize homogeneous Riemann manifolds and determine the structures of Killing vector fields by the knowledge of their minimal differential polynomials.

\section{§ 4. Embeddability of algebraic Riemann manifolds}

We shall prove the following "embeddability by general scalar curvatures":

Proposition 4.1. Let $M$ be a compact Riemann manifold of class $C^{\omega}$. Assume that $M$ is nowhere homogeneous in the sense that for any distinct points $p, q$ of $M$, there exists no $C^{\omega}$ isometry $h, h(p)=q$, of a neighborhood of $p$ onto a neighborhood of $q$. Then there exist $C^{\omega}$ functions $f_{1}, \cdots, f_{N}$, with sufficiently large $N$, such that

(i) each $f_{i}$ is a general scalar curvature, i.e. $f_{i}$ is expressed as a linear combination with constant coefficients of contractions of the curvature tensor and its covariant differentials up to some order, and

(ii) the mapping $\left(f_{1}, \cdots, f_{N}\right): M \rightarrow \boldsymbol{R}^{N}$ is an embedding (not isometric) of $M$.

From this proposition we have at once

THEOREM 4.2. Let $M$ be a compact Riemann manifold of class $C^{\Omega}$. Assume that $M$, viewed as a $C^{\omega}$ Riemann manifold, is nowhere homogeneous. Then $M$ admits a $C^{\Omega}$ embedding in some vector space $\boldsymbol{R}^{N}$.

Example 4.3. There exist $C^{\Omega}$ Riemann manifolds without any nonconstant $C^{\Omega}$ function. Indeed, let $\boldsymbol{T}_{\theta}^{n}$ be an $n$-dimensional torus with the 
$C^{\Omega}$ structure and the $C^{\Omega}$ Riemann metric such that the covering mapping $\pi: \boldsymbol{R}^{n} \rightarrow \boldsymbol{T}_{\theta}^{n}=\boldsymbol{R}^{n} / \boldsymbol{Z}^{n}$ is of class $C^{\Omega}$ and locally isometric, where $\boldsymbol{R}^{n}$ is equipped with the Euclidean metric. The argument in Palais [8, p. 63] shows that $\boldsymbol{T}_{\theta}^{n}$ has no non-constant $C^{a}$ function. Furthermore, using this fact, we know that every $C^{\Omega}$ tensor field on $\boldsymbol{T}_{\theta}^{n}$ is parallel and any $C^{\Omega}$ diffeomorphism of $\boldsymbol{T}_{\theta}^{n}$ is affine.

As another consequence of Proposition 4.1 we obtain

Corollary 4.4. Let $M, \bar{M}$ be compact Riemann manifolds of class $C^{\Omega}$. Assume that $M$, viewed as a $C^{\omega}$ Riemann manifold, is nowhere homogeneous, and assume that there exists a $C^{\omega}$ isometry $h$ of $M$ onto $\bar{M}$. Then $h$ is of class $C^{\Omega}$, and hence $M, \bar{M}$ are $C^{\Omega}$ isometric.

Proof. By Proposition 4.1 we note that every point of $M$ has $a$ coordinate neighborhood with local coordinate system consisting of general scalar curvatures. Since isometries preserve general scalar curvatures, our corollary is now obvious.

We note that there exist $C^{a}$ Riemann manifolds $M, \bar{M}$ which are $C^{0}$ and hence $C^{\omega}$ isometric ([5, p. 169]), but are never $C^{\Omega}$ diffeomorphic. Indeed, let $\boldsymbol{T}_{\theta}^{1}$ be the one-dimensional $C^{\Omega}$ Riemann manifold in Example 4.3 , and let $S^{1}=\left\{(x, y) \mid x^{2}+y^{2}=(1 / 2 \pi)^{2}\right\}$ be the $C^{\Omega}$ submanifold of the Euclidean space $R^{2}$, with the induced metric. Clearly, $T_{\theta}^{1}$ and $S^{1}$ are isometric, but not $C^{\Omega}$ diffeomorphic.

In order to prove Proposition 4.1, it is convenient to introduce the notion of natural functions on Riemann manifolds.

Let $V$ be an Euclidean vector space of dimension $n$ and let $O(V)$ be the group of orthogonal transformations of $V$. Fix a non-negative integer $m$. We consider the real vector space

$$
\widetilde{\mathscr{R}}=\bigoplus_{k=0}^{m}\left(\otimes^{k+4} V\right)^{*}
$$

the direct sum of the dual vector spaces $\left(\otimes^{k+4} V\right)^{*}$ of the $(k+4)$-th tensor powers of $V$. The group $O(V)$ acts on $\widetilde{R}$ (on the right) as follows. Let $a \in O(V)$. First, for $S \in\left(\otimes^{s} V\right)^{*}$, define $S^{a} \in\left(\otimes \otimes^{s} V\right)^{*}$ by the formula

$$
S^{a}\left(x_{1} \otimes \cdots \otimes x_{s}\right)=S\left(a x_{1} \otimes \cdots \otimes a x_{s}\right) .
$$

Then, for $\tilde{R}=\left(R_{0}, R_{1}, \cdots, R_{m}\right) \in \widetilde{\mathscr{R}}, R_{k} \in\left(\otimes^{k+4} V\right)^{*}$, we define $\tilde{R}^{a} \in \widetilde{\mathscr{R}}$ to be

$$
\tilde{R}^{a}=\left(R_{0}^{a}, R_{1}^{a}, \cdots, R_{m}^{a}\right) .
$$


We denote by $R[\widetilde{R}]^{O(V)}$ the algebra of $O(V)$-invariant polynomial functions $\phi: \widetilde{R} \rightarrow \boldsymbol{R}$, i.e. polynomial functions $\phi$ such that $\phi\left(\tilde{R}^{a}\right)=\phi(\tilde{R})$ for all $\tilde{R} \in \widetilde{R}$ and $\mathrm{a} \in O(V)$.

Now, let $M$ be a $C^{\infty}$ Riemann manifold of dimension $n$, and $f: M \rightarrow \boldsymbol{R}$ a $C^{\infty}$ function. We shall say that $f$ is a natural function if there exists an $O(V)$-invariant polynomial function $\phi \in R[\widetilde{R}]^{o(V)}$ such that

$$
f(x)=\phi\left(R_{x}, R_{x}^{\prime}, \cdots, R_{x}^{(m)}\right)
$$

for all $x \in M$, where $R_{x}^{(k)}, k=0, \cdots, m$, denote the $k$-th covariant differentials $\left(\nabla^{k} R\right)_{x} \in\left(\otimes^{k+4} T_{x}(M)\right)^{*}$ of the Riemann curvature tensor $R$, and each tangent space $T_{x}(M)$ is identified with $V$ by a linear isometry. Clearly, if $M$ is a Riemann manifold of class $C^{\omega}$ (resp. $C^{\Omega}$ ), then natural functions on $M$ are of class $C^{\omega}$ (resp. $C^{\Omega}$ ). If $M$ is locally homogeneous, then constant functions are the only natural functions on $M$.

Next, let us recall some notions in Kobayashi-Nomizu [5, Vol. I]. Let $M$ be a $C^{\infty}$ Riemann manifold of dimension $n$. Let $\pi: O(M) \rightarrow M$ be the bundle of orthonormal frames over $M$. For $u \in O(M)$ and an orthogonal matrix $a \in O(n)$, the right action of $O(n)$ on $O(M)$ defines the orthonormal frame $R_{a} u=u a$. Each $A \in \mathfrak{D}(n)$, the Lie algebra of $O(n)$, defines the fundamental vector field $A^{*}$ and each $\xi \in \boldsymbol{R}^{n}$ defines the standard horizontal vector field $B(\xi)$.

In our proof, for a tensor field $S$ of type $(0, s)$, we shall consider instead of $S$ itself the functions $g_{\alpha}: O(M) \rightarrow R$ defined by the formulas

$$
g_{\alpha}(u)=S\left(X_{\sigma_{1}}, \cdots, X_{\alpha_{s}}\right), \quad u=\left(X_{1}, \cdots, X_{n}\right) \in O(M),
$$

where $\alpha=\left(\alpha_{1}, \cdots, \alpha_{s}\right)$ are $s$-tuples of positive integers $\leqq n$. It is easy to verify that the functions $g_{\alpha}$ satisfy the following formulas.

LEMMA 4.5.

(i ) $R_{a}^{*} g_{\alpha}=\sum_{i_{1}, \ldots, i_{s}} a_{\alpha_{1}}^{i_{1}} \cdots a_{\alpha_{s}}^{i_{s}} g_{i_{1} \cdots i_{s}}$ for each $a=\left(a_{j}^{i}\right) \in O(n)$, where $R_{a}^{*} g_{\alpha}$ : $O(M) \rightarrow \boldsymbol{R}$ is defined by $\left(R_{a}^{*} g_{\alpha}\right)(u)=g_{\alpha}(u a)$.

(ii) $A^{*} g_{\alpha}=\sum_{i_{1}} A_{\alpha_{1}}^{i_{1}} g_{i_{1} \alpha_{2} \cdots \alpha_{s}}+\cdots+\sum A_{\alpha_{s}}^{i_{s}} g_{\alpha_{1} \cdots \alpha_{s-1} i_{s}}$ for each $A=\left(A_{j}^{i}\right)$ $\in \mathfrak{o}(n)$.

(iii) $B(\xi) g_{\alpha}=\sum_{i} \xi^{i} g_{\alpha ; i}$ for each $\xi=\left(\xi^{i}\right) \in \boldsymbol{R}^{n}$, where $g_{\alpha ; i}(u)=(\nabla S)\left(X_{\alpha_{1}}, \cdots, X_{\alpha_{s}} ; X_{i}\right), u=\left(X_{1}, \cdots, X_{n}\right)$.

In order to prove Proposition 4.1 we need another lemma.

LemMa 4.6. Let $M$ be a $C^{\omega}$ Riemann manifold of dimension $n$, and 
$R$ its curvature tensor, viewed as tensor field of type (0,4). For each $(k+4)$-tuple $\alpha=\left(\alpha_{1}, \cdots, \alpha_{k+4}\right)$ with $\alpha_{1}, \cdots, \alpha_{k+4} \in\{1, \cdots, n\}$, we define $f_{\alpha}: O(M) \rightarrow R$ by the formula

$$
f_{\alpha}(u)=\left(\nabla^{k} R\right)\left(X_{\alpha_{1}}, \cdots, X_{\alpha_{4}} ; X_{\alpha_{5}} ; \cdots ; X_{\alpha_{k+4}}\right), \quad u=\left(X_{1}, \cdots, X_{n}\right) \in O(M) .
$$

Assume that $M$ is nowhere homogeneous as in Proposition 4.1. Let $u \in$ $O(M)$, and let $D \in T_{u}(O(M))$ be a non-zero tangent vector. Then, there exists a non-negative integer $m$ and an $(m+4)$-tuple $\beta=\left(\beta_{1}, \cdots, \beta_{m+4}\right)$ with $\beta_{1}, \cdots, \beta_{m+4} \in\{1, \cdots, n\}$ such that the derivative $D\left(f_{\beta}\right)$ at $u$ of the function $f_{\beta}$ does not vanish.

Proof. The tangent vector $D$ can be expressed as

$$
D=A_{u}^{*}+B(\xi)_{u}
$$

with some $A \in \mathfrak{D}(n)$ and $\xi \in \boldsymbol{R}^{n}$, because the fundamental vector fields and the standard horizontal vector fields give an absolute parallelism in $O(M)$. Let $u_{t}$ be the integral curve for the vector field $A^{*}+B(\xi)$ such that $u=u_{0}$. Note that for each $\alpha=\left(\alpha_{1}, \cdots, \alpha_{k+4}\right)$ the function $f_{\alpha}\left(u_{t}\right)$ of $t$ is of class $C^{\omega}$ and satisfies

$$
\left(\frac{d}{d t}\right)_{t=0}^{l} f_{\alpha}\left(u_{t}\right)=D\left\{\left(A^{*}+B(\xi)\right)^{l-1} f_{\alpha}\right\}
$$

for any $l \geqq 1$. Furthermore, using the formulas (ii), (iii) of Lemma. 4.5, we see that the function $\left(A^{*}+B(\xi)\right)^{l-1} f_{\alpha}$ is expressed as a linear combination (with constant coefficients) of the functions $f_{\beta}$, where $\beta$ are $m$-tuples of integers in $\{1, \cdots, n\}, k+4 \leqq m \leqq k+4+l-1$. We contend that there exists $\alpha=\left(\alpha_{1}, \cdots, \alpha_{k+4}\right)$ with some $k$, and an integer $l \geqq 1$ such that $\left(\frac{d}{d t}\right)_{t=0}^{l} f_{\alpha}\left(u_{t}\right) \neq 0$. This will show that $D\left(f_{\beta}\right) \neq 0$ for some $\beta=\left(\beta_{1}, \cdots, \beta_{m}\right)$, $k+4 \leqq m \leqq k+l+3$.

To prove our contention, suppose that for any $\alpha=\left(\alpha_{1}, \cdots, \alpha_{k+4}\right)$ and any integer $l \geqq 1$, we had $\left(\frac{d}{d t}\right)_{t=0}^{l} f_{\alpha}\left(u_{t}\right)=0$. Then for any $\alpha$ and any $t$, we would have $f_{\alpha}\left(u_{t}\right)=f_{\alpha}\left(u_{0}\right)$, and hence by Theorem 7.2 of [5, Vol. I], for each $t$ we would have a $C^{\omega}$ isometry of a neighborhood of $\pi\left(u_{0}\right)$ onto a neighborhood of $\pi\left(u_{t}\right)$. This contradicts the nowhere-homogeneity of $M$. Lemma 4.6 is proved.

Proof of Proposition 4.1. We have to investigate more closely the functions $f_{\alpha}$ of Lemma 4.6. For a non-negative integer $k$, we denote by 
$I_{k+4}$ the set of all $(k+4)$-tuples $\alpha=\left(\alpha_{1}, \cdots, \alpha_{k+4}\right)$ such that $\alpha_{1}, \cdots, \alpha_{k+4} \in$ $\{1, \cdots, n\}$. Let $m$ be a non-negative integer. Put $I=\bigcup_{0 \leqq k \leqq m} I_{k+4}$ and let $\left\{\boldsymbol{R}_{\alpha}\right\}_{\alpha \in I}$ be a family of $\boldsymbol{R}_{\alpha}=\boldsymbol{R}$, indexed by $I$. To define a right action of $O(n)$ on the vector space $\prod_{\alpha \in I} \boldsymbol{R}_{\alpha}$, we introduce the following notation. For $a=\left(a_{j}^{i}\right) \in O(n)$ and $\alpha=\left(\alpha_{1}, \cdots, \alpha_{k+4}\right) \in I_{k+4}, \beta=\left(\beta_{1}, \cdots, \beta_{l+4}\right) \in I_{l+4}$, we put

$$
\begin{aligned}
& a_{\beta}^{\alpha}=0 \quad \text { if } k \neq l, \\
& a_{\beta}^{\alpha}=a_{\beta_{1}}^{\alpha_{1}} a_{\beta_{2}}^{\alpha_{2}} \cdots a_{\beta_{k+4}}^{\alpha_{k+4}}
\end{aligned} \quad \text { if } k=l .
$$

For $a=\left(a_{j}^{i}\right) \in O(n)$ and $x=\left(x_{\alpha}\right)_{\alpha \in I} \in \prod_{\alpha \in I} \boldsymbol{R}_{\alpha}$, we define $R_{\otimes a} x \in \prod_{\alpha \in I} \boldsymbol{R}_{\alpha}$ by the formulas

$$
\left(R_{\otimes a} x\right)_{\alpha}=\sum_{\beta \in I} a_{\alpha}^{\beta} x_{\beta}
$$

We can now prove our proposition. Consider the mapping

$$
f: O(M) \rightarrow \prod_{\alpha \in I} R_{\alpha}
$$

defined by

$$
\boldsymbol{f}(u)=\left(f_{\alpha}(u)\right)_{\alpha \in I},
$$

where $f_{\alpha}(u)=\left(\nabla^{k} R\right)\left(X_{\alpha_{1}}, \cdots, X_{\alpha_{k+4}}\right), u=\left(X_{1}, \cdots, X_{n}\right)$ as in Lemma 4.6. By Lemma 4.5, (i), we see that the mapping $f: O(M) \rightarrow \prod_{\alpha \in I} \boldsymbol{R}_{\alpha}$ is equivariant, i.e. $f \circ R_{a}=R_{\otimes a} \circ f$ for any $a \in O(n)$. We contend that if $m$ is large enough, then $f$ is an embedding. Indeed, using Lemma 4.6 and the compactness of $O(M)$ we see that $f$ is an immersion for large $m$. Repeating a similar argument and taking larger $m$ we can make $f$ injective. Our contention is proved.

To get our desired embedding of $M$ we use the action of $O(n)$ on $O(M)$ and the integrations on the orbits. To be more precise, let $p \in M$ and consider the fibre $\pi^{-1}(p) \subset O(M)$ over $p$. The image $f\left(\pi^{-1}(p)\right) \subset \prod_{\alpha \in I} \boldsymbol{R}_{\alpha}$ is a compact submanifold of codimension, say $d$. Let $\nu$ be its tubular neighborhood. By means of the action of $O(n)$ we have a submersion $\chi: \nu \rightarrow \boldsymbol{R}^{d}$ such that

$$
\chi\left(R_{\otimes a} x\right)=\chi(x)
$$

for any $x \in \nu$ and any $a \in O(n)$. By the Weierstrass approximation theorem we get a polynomial mapping

$$
\Phi: \prod_{\alpha \in I} \boldsymbol{R}_{\alpha} \rightarrow \boldsymbol{R}^{d}
$$


such that $\Phi$ is $C^{1}$-close to $\chi$ in a neighborhood of $f\left(\pi^{-1}(p)\right)$. Integrating each component of $\Phi$ over $O(n)$ in the usual way, we obtain a polynomial mapping

$$
\bar{\Phi}: \prod_{\alpha \in I} \boldsymbol{R}_{\alpha} \rightarrow \boldsymbol{R}^{d}
$$

having the following properties.

(i ) $\bar{\Phi}$ is $O(n)$-invariant, i.e. $\bar{\Phi} \circ R_{\otimes a}=\bar{\Phi}$ for any $a \in O(n)$,

(ii) $\bar{\Phi}$ is a submersion in a neighborhood of $\boldsymbol{f}\left(\pi^{-1}(p)\right)$, and hence

(iii) for any $x \in \boldsymbol{f}\left(\pi^{-1}(p)\right)$, the normal space to $f\left(\pi^{-1}(p)\right)$ at $x$ is isomorphic to $T_{\bar{\phi}(x)}\left(\boldsymbol{R}^{d}\right)$ by the induced mapping $\bar{\Phi}_{*}$. Thus the composite mapping $\bar{\Phi} \circ \boldsymbol{f}: O(M) \rightarrow \boldsymbol{R}^{d}$ gives a well-defined mapping

$$
F: M \rightarrow R^{d}
$$

such that $F \circ \pi=\bar{\Phi} \circ f$. It is easy to see that $F$ gives rise to an embedding in a neighborhood of $p$, and that each component of $F$ is a natural function.

In this way, for each point $p^{\prime}$ of $M$ we can construct a mapping $F^{\prime}: M \rightarrow R^{d}$ such that components of $F^{\prime}$ are natural functions, and $F^{\prime}$ gives rise to an embedding in a neighborhood of $p^{\prime}$. Hence, by the compactness of $M$ we get an immersion

$$
\begin{aligned}
\boldsymbol{F}=\left(F, F^{\prime}, \cdots\right): & M \rightarrow \boldsymbol{R}^{d} \oplus \cdots \oplus \boldsymbol{R}^{d} \\
& \quad \text { (direct sum taken sufficiently many times). }
\end{aligned}
$$

By a similar argument as above we can make $\boldsymbol{F}$ injective further. On the other hand, by the fundamental theorem of invariant theory for $O(n)$ we see that every natural function on $M$ is expressed as a linear combination of contractions of $R$ and its covariant differentials (see, for example, [1], [2]). Therefore, $\boldsymbol{F}$ gives the desired embedding.

\section{REFERENCES}

[1] M. Atiyah, R. Bott and V. K. Patodi, On the heat equation and the index theorem, Invent. Math., 19 (1973), 279-330.

[2] M. Berger, P. Gauduchon and E. Mazet, Le spectre d'une variété riemannienne, Lecture Notes in Math., 194, Springer, 1971.

[ 3 ] É. Cartan, Leçons sur la géométrie des espaces de Riemann, Gauthier-Villars, 1946.

[4] J. Cheeger and D. Ebin, Comparison Theorems in Riemannian Geometry, NorthHolland Publ., 1975.

[5] S. Kobayashi and K. Nomizu, Foundations of Differential Geometry, Interscience, 
1963 (Vol. I), 1969 (Vol. II).

[6] J. Nash, Real algebraic manifolds, Ann. of Math., 56 (1952), 405-421.

[ 7 ] K. Nomizu, Sur les algèbres de Lie de générateurs de Killing et l'homogénéité d'une variété Riemannienne, Osaka J. Math., 14 (1962), 45-51.

[ 8 ] R. Palais, Equivariant real algebraic differential topology, Part I, Smoothness categories and Nash manifolds, Notes, Brandeis Univ., 1972.

[ 9 ] M. Shiota, Nash manifolds, Lecture Notes in Math., 1269, Springer, 1987.

[10] I. M. Singer, Infinitesimally homogeneous spaces, Comm. Pure Appl. Math., 13 $(1960), 685-697$.

Department of Mathematics

College of General Education

Nagoya University

Chikusa-ku, Nagoya 464

Japan 